Revue d'histoire de l'Amérique française

REVUE D.HISTOIRE DE L'AMÉRIQUE FRANÇAISE

\title{
Penser le Canada
}

\section{La mise en place des assises intellectuelles de l'État canadien moderne (1838-1840)}

\section{Michel Ducharme}

Volume 56, numéro 3, hiver 2003

URI : https://id.erudit.org/iderudit/007618ar

DOI : https://doi.org/10.7202/007618ar

Aller au sommaire du numéro

\section{Éditeur(s)}

Institut d'histoire de l'Amérique française

\section{ISSN}

0035-2357 (imprimé)

1492-1383 (numérique)

Découvrir la revue

\section{Citer cet article}

Ducharme, M. (2003). Penser le Canada : la mise en place des assises intellectuelles de l'État canadien moderne (1838-1840). Revue d'histoire de l'Amérique française, 56(3), 357-386. https://doi.org/10.7202/007618ar

\section{Résumé de l'article}

L’État étant une idée (Burdeau, 1970, 14), nous devons comprendre les fondements intellectuels qui sous-tendent son existence. L'État canadien tel qu'il est défini dans la seconde moitié du XIX ${ }^{\mathrm{e}}$ siècle apparaît, du point de vue des provinces centrales, comme le fruit d'un compromis politique et idéologique entre divers groupes et tirant sa genèse de la période allant de 1838 à 1840. Bien que l'historiographie les ait négligées, ces années constituent le creuset duquel émerge le Canada moderne. À cette époque charnière où l'espace canadien doit être redéfini vu les graves troubles politiques coloniaux, les intellectuels, les politiciens et les groupes de pression explicitent leur vision de l'avenir des deux Canadas et proposent leurs solutions à l'impasse constitutionnelle. À partir des définitions théoriques du libéralisme et du nationalisme, nous tâchons de mettre en lumière les points de rencontre entre les divers intervenants (tory ou réformistes, coloniaux ou métropolitains). Ces éléments laissent entrevoir les bases sur lesquelles s'édifiera le Canada. 


\section{Penser le Canada \\ La mise en place des assises intellectuelles de l'État canadien moderne (I838-I840 $)^{1}$

\author{
MICHEL DUCHARME \\ Département d'histoire \\ Université McGill
}

RÉSUMÉ - L'État étant une idée (Burdeau, 1970, 14), nous devons comprendre les fondements intellectuels qui sous-tendent son existence. L'État canadien tel qu'il est défini dans la seconde moitié du XIX siède apparaît, du point de vue des provinces centrales, comme le fruit d'un compromis politique et idéologique entre divers groupes et tirant sa genèse de la période allant de 1838 à 1840 . Bien que l'historiographie les ait négligées, ces années constituent le creuset duquel émerge le Canada moderne. A cette époque charnière où l'espace canadien doit être redéfini vu les graves troubles politiques coloniaux, les intellectuels, les politiciens et les groupes de pression explicitent leur vision de l'avenir des deux Canadas et proposent leurs solutions à l'impasse constitutionnelle. A partir des définitions théoriques du libéralisme et du nationalisme, nous tâchons de mettre en lumière les points de rencontre entre les divers intervenants (tory ou réformistes, coloniaux ou métropolitains). Ces éléments laissent entrevoir les bases sur lesquelles s'édifiera le Canada.

ABSTRACT - The State being an idea (Burdeau, 1970, 14), we must try to understand the intellectual foundations that structure its existence. For the Central Provinces, the advent of the Canadian State in the second half of the Nineteenth Century appeared as the result of a political and ideological compromise between many different groups. It represented the successful conclusion of a long negotiation which began in 1838-1840.

1. Cet article est tiré de notre mémoire de maitrise intitulé Du triptyque idéologique. Libéralisme, nationalisme et impérialisme au Haut-Canada, au Bas-Canada et en Grande-Bretagne entre 1838 et 1840, mémoire de maîtrise (histoire), Université de Montréal, 1999, 192 p. La rédaction de cet article a été rendue possible grâce à l'aide financière du CRSH et du FCAR. Nous voulons remercier Michèle Dagenais qui a dirigé le mémoire et Brian Young qui a commenté une première version du texte. 
Though historiography has neglected these years, the modern Canadian State emerged during this time. With reform being necessary to overcome the constitutional deadlock after the Rebellions, intellectuals and politicians expressed their views on the future of British North America. Basing our research on theoretical definitions of liberalism and nationalism, we try to illustrate the similarities between the many discourses (tory or reformist, colonial or metropolitan) on which the foundations of Canada were laid.

L' ANNÉE I837 MARQUE Un TOURNANT dans l'histoire canadienne. Cette année-là, plusieurs crises se conjuguent et engendrent des insurrections dans les deux Canadas. Au Bas-Canada, l'Assemblée législative étant sous la mainmise d'une majorité de députés appartenant au parti rebelle, la constitution est suspendue en février 1838. Jusqu'à l'union des Canadas en février 1841, la colonie est administrée par un gouverneur assisté d'un conseil spécial ${ }^{2}$. C'est à cette administration extraordinaire qu'incombe la tâche de mater la seconde rébellion bas-canadienne de novembre 1838 et de veiller au retour du calme dans la colonie. Au Haut-Canada, les institutions politiques régulières, dominées par les tories, participent au processus de "pacification».

L'historiographie a généralement banalisé les années 1838-1840 afin d'arriver rapidement à l'Acte d'Union, jalon de l'histoire politique et constitutionnelle. Entre 1837 et 1840, l'étude de la dynamique politique coloniale perd ses adeptes comme si, une fois les radicaux déchus, rien ne méritait plus l'attention, ni débats ni enjeux. De toutes les synthèses publiées portant sur les colonies pendant ces années, seules celles de Gerald Craig, Upper Canada. The Formative Years 1784-1841, et de Jacques Monet, The Last Cannon $S h t^{3}$, abordent de manière globale la période. Sans délaisser le rapport Durham, Craig analyse l'évolution de la politique dans la province supérieure, le rôle des tories et la renaissance des modérés. De la même manière, Monet étudie ce qui se passe au Bas-Canada. Il s'attarde principalement aux débats entre les intellectuels et les politiciens non discrédités par les rébellions. Plus généralement et hormis quelques études spécialisées, les travaux historiques effleurent cette courte période en portant leur attention presque exclusivement sur la poursuite de l'agitation insurrectionnelle et sur

2. Pour une étude du Conseil spécial, voir Stephen Watt, Authoritarianism, Constitutionalism and the Special Council of Lower Canada, 1838-1840, mémoire de maîtrise (histoire), Université McGill, 1997, 149 p.

3. Gerald Craig, Upper Canada. The Formative Years 1784-1841 (Toronto, McClelland \& Stewart, 1963), 252-275 ; Jacques Monet, The Last Cannon Shot (Toronto, University of Toronto Press, 1969), 1-57. Pour la version française, voir La première révolution tranquille (Montréal, Fides, 1983). 
le rapport de lord Durham ${ }^{4}$. Ce faisant, l'historiographie a passé sous silence l'enjeu véritable de la période.

Les années 1838-1840 apparaissent essentielles à l'histoire canadienne parce qu'elles constituent le creuset duquel émerge le Canada moderne. Il ne s'agit pas de prétendre que le Canada apparaît définitivement à cette date ni que son avenir est inéluctable, ce serait oublier les trente années de négociation entre les rébellions et la Confédération. Néanmoins, par leur échec, les rébellions ont légué un cadre de référence à l'intérieur duquel l'État canadien s'est développé, cadre qui influe encore sur la vie de la fédération. Jusqu'à présent, les historiens du «State formation» ont abordé la question de la formation de l'État canadien en étudiant la mise en place de nouvelles institutions, de nouvelles formes et de nouveaux mécanismes de gouvernance après 1840 . S'inspirant des travaux de Philip Abrams, Philip Corrigan, Derek Sayer et parfois même de Michel Foucault, ces historiens ont mis en lumière la «révolution culturelle» qui a affecté le Canada au milieu du XIX ${ }^{\mathrm{e}}$ siècle et permis l'avènement d'un nouvel "ordre libéral». Toute la richesse de ce courant historiographique n'a pas encore été épuisée $e^{5}$. Les institutions créées à ce moment par l'État lui permettent sans aucun doute de redéfinir les relations sociales dans la colonie. Par

4. Pensons à Donald Creighton, The Empire of St. Lawrence (Toronto, MacMillan, 1956) (1937), 321-348; Allan Greer, The Patriots and the People. The Rebellion of 1837 in Rural Lower Canada (Toronto, University of Toronto Press, 1993), 332-363; Fernand Ouellet, Histoire socio-économique du Québec, 1750-1850 (Montréal, Fides, 1966), 413-440; Colin Read, The Rising in Western Upper Canada, 1837-1838: The Duncombe Revolt and After (Toronto, University of Toronto Press, 1982), 107-163 (et la liste pourrait s'allonger).

5. Pour les travaux théoriques, consulter: Philip Abrams, «Notes on the Difficulty of Studying the State", Journal of Historical Sociology, 1,1 (1988) : 57-84; Philip Corrigan et Derek Sayer, The Great Arch : English State Formation as Cultural Revolution (Oxford, Basil Blackwell, 1985), 268 p.; Michel Foucault, "Gouvernmentality", dans Graham Burchell, dir. et al, The Foucault Effect : Studies in Governmentality (Chicago, University of Chicago Press, 1991), 87-104; Surveiller et punir : naissance de la prison (Paris, Gallimard, 1975), 318 p.; Colin Gordon, dir., Power/Knowledge: Selected Interviews and Other Writings, 1972-1977 (Brighton, Harvester Press, 1980), 270 p. Pour l'application de la thèse du «State formation» dans le cadre canadien, voir : Bruce Curtis, The Politics of Population. State Formation, Statistics, and the Census of Canada, 1840-1875 (Toronto, University of Toronto Press, 2001), 385 p.; True Government by Choice Men? Inspection, Education and the State Formation in Canada West (Toronto, University of Toronto Press, 1992), 250 p.; Jean-Marie Fecteau, Un nouvel ordre des choses : la pauvreté, le crime, l'État au Québec, de la fin du XVIII ${ }^{e}$ siècle à 1840 (Outremont, VLB éditeur, 1989), 231 p.; Brian Young, The Politics of Codification. The Lower Canadian Civil Code of 1866 (Montréal, The Osgoode Society for Canadian Legal History/McGillQueen's University Press, 1994), 264 p. Pour les perspectives d'avenir de ce courant historiographique, lire : Ian McKay, «The Liberal Order Framework : A Prospectus for a Reconnaissance of Canadian History", Canadian Historical Review, 81,4 (décembre 2000) : 617-645. 
l'analyse de cet effort de restructuration et des motivations qui la soustendent, nous découvrons bel et bien la mise en place d'un nouvel ordre social. Néanmoins, les principes politiques qui rendent l'État légitime sont rarement discutés. Cette absence est d'autant plus surprenante que l'État apparaît lui-même comme une légitimation intellectuelle de l'organisation du pouvoir, comme une conceptualisation des notions d'autorité, d'ordre et de hiérarchie au sein de la société. L'État moderne peut façonner les relations sociales à la condition d'être préalablement entouré d'une aura de légitimité. De cette conceptualisation du pouvoir légitime naissent ensuite les institutions constitutionnelles, politiques et régulatrices qui encadrent la société. Et ce sont par ces institutions que l'État impose sa volonté. Partant de la prémisse que «l'État est, au sens plein du terme, une idée $e^{6}$, il s'avère primordial de retrouver les fondements intellectuels qui soustendent son existence. Retrouver les assises politiques et intellectuelles du Canada, c'est se donner les moyens de le comprendre.

L'avènement de l'État canadien moderne apparaît, du point de vue des provinces centrales, comme le fruit d'un compromis complexe qui tire sa genèse des années 1838-1840. C'est à ce moment que les principes sur lesquels l'État canadien va s'échafauder deviennent centraux dans les discours. Malgré des différences idéologiques entre les divers groupes coloniaux et métropolitains intéressés par l'avenir du Canada, certains points communs se dégagent. Le Canada moderne se développera au sein de l'Empire britannique en suivant les principes libéraux tels qu'ils ont cours en Grande-Bretagne et dans le cadre d'une coopération entre Canadiens français et Canadiens anglais.

Pour comparer les principes et les idées inclus dans les discours, il est d'abord nécessaire de clarifier certains concepts. Les théories libérale et nationale, souvent utilisées mais rarement définies, doivent être mieux cernées, ce que nous ferons en nous servant de la littérature de l'époque ainsi que de travaux d'universitaires. Ensuite, nous traiterons des idées présentes dans la métropole et dans les colonies. Notre corpus se compose de documents produits ou ayant été utilisés entre le $1^{\text {er }}$ janvier 1838 et le $1^{\text {er }}$ janvier 1840. L'impossibilité de dépouiller de manière systématique toutes les sources disponibles nous a conduit à sélectionner les documents en fonction de deux critères. Premièrement, ils doivent exprimer le point de vue de factions «extrémistes» se trouvant à l'intérieur du spectre

6. Georges Burdeau, L'État (Paris, Seuil, 1970), 14. Une idée similaire est développée par Craig Calhoun dans Nationalism (Buckingham, Open University Press, 1997), 10. 
idéologique reconnu légalement. Deuxièmement, ils doivent avoir une pertinence en eux-mêmes. C'est ainsi que nous utilisons des documents métropolitains. Les décisions politiques importantes relevant de Londres, l'histoire canadienne doit être remise dans le contexte impérial. Dans cette perspective, nous analyserons des textes tels que les dépêches de lord Russell et le Report on the Affairs of British North America de lord Durham. Par ailleurs, les discours coloniaux haut et bas-canadiens doivent être traités de manière complémentaire, le sort des deux colonies étant intimement lié. Pour aborder les positions bas-canadiennes, nous faisons appel aux journaux, et notamment à la feuille Le Canadien de Québec. Son directeur, Étienne Parent, fut amené, au lendemain des rébellions, à assumer le rôle de représentant des aspirations canadiennes-françaises et à défendre les intérêts de ses compatriotes. De tous les hommes publics canadiensfrançais influents encore en action entre 1838 et 1840, Parent apparaît comme le plus réformiste et le plus nationaliste. Pour retrouver les valeurs des tories bas-canadiens, nous nous appuierons sur les éditoriaux de la Montreal Gazette, "principal journal tory du Bas-Canada ${ }^{7}$ ». Pour traiter de l'idéologie des réformistes du Haut-Canada, nous retiendrons la proposition de Robert Baldwin sur le gouvernement responsable. Quant à la position tory de cette province, elle sera mise en évidence dans des textes émanant de la Législature haut-canadienne.

\section{I - S'ASSURER UNE BASE DE COMPARAISON : LA DÉFINITION DES CONCEPTS}

\section{I.I Le libéralisme ou comment concilier liberté et ordre}

Le concept de «libéralisme» a reçu diverses acceptions dans l'historiographie canadienne. Parmi les plus récentes, il y a celles des tenants de l'approche du «State formation», qui empruntent une grille d'analyse tantôt marxisante, tantôt proche des thèses de Theda Skocpol. Leur attention porte sur l'instauration d'un ordre libéral à l'intérieur duquel les individus doivent apprendre à s'autoréguler. Le développement des institutions municipales et scolaires incarne cette volonté de contrôler la société par la voix du consentement. La liberté n'est alors possible que grâce à l'intériorisation des nouvelles normes sociales ${ }^{8}$. Pour sa part, le libéralisme d'Yvan Lamonde est

7. George L. Parker, "Armour, Robert», Dictionnaire biographique du Canada (Sainte-Foy, Les Presses de l’Université Laval, 1985), $8: 24$.

8. Dans l'introduction de l'ouvrage Colonial Leviathan, les directeurs estiment que leur livre remet en cause "the liberal myth of the liberal State», Allan Greer et Ian Radforth, dir., Colonial Leviathan (Toronto, University of Toronto Press, 1992), 13. 
progressif, réformateur et démocratique. Les décennies suivant les rébellions apparaissent marquées par l'échec du projet libéral et non par sa réussite. Alors que Lamonde assimile plus ou moins libéralisme et démocratie, Janet Ajzenstat soutient que, dans la première moitié du $\mathrm{xIx}^{\mathrm{e}}$ siècle, ces deux idéologies ne sont pas synonymes. Les libéraux préfèrent des institutions mixtes (monarchie, aristocratie et démocratie) pour éviter la mise en place d'une tyrannie démocratique ${ }^{9}$. Si Ajzenstat a démontré la parenté intellectuelle entre lord Durham et John Beverley Robinson, Rod Preece est allé plus loin en présentant les tories canadiens comme des conservateurs à la Edmund Burke. Or, ce conservatisme burkien ne serait qu'une variante du libéralisme classique ${ }^{10}$. Face à autant d'interprétations, un retour aux sources du libéralisme est non seulement souhaitable mais nécessaire.

En fait, le libéralisme constitue une idéologie éclatée. Il englobe aussi bien la théorie du droit naturel que celle de l'utilité. Il inclut la théorie du contrat social, bien que certains libéraux la répudient et que des non-libéraux l'utilisent. Pour certains, la souveraineté appartient au Parlement; pour d'autres, à la nation. Le terme qualifie aussi deux réalités distinctes. Outre l'idéologie généreuse et universelle consacrée à la recherche de la liberté, le libéralisme est également pratique : il s’incarne dans l’État libé$\mathrm{ral}^{11}$. Dans ce cas, il travaille à sa propre conservation. De dynamique, il devient progressivement conservateur.

Malgré la diversité du libéralisme, certains principes généraux font consensus. L'idéologie libérale se structure autour de l'individu. Toutefois,

9. Yvan Lamonde, Histoire sociale des idées au Québec 1760-1896 (Montréal, Fides, 2000), 283379 ; Janet Ajzenstat, «Modern Mixed Government : A Liberal Defence of Inequality ", Canadian Journal of Political Science, 18,1 (mars 1985) : 119-134; The Political Thought of Lord Durham (Montréal, McGill-Queen's University Press, 1988), 137 p. Il faut toutefois remarquer que, dans son Histoire sociale des idées, 329, Yvan Lamonde mentionne que les conservateurs partageaient une forme de libéralisme modéré sans développer plus avant cette idée. Dans un article récent, il propose d'étudier ce libéralisme modéré dominant : voir "La vie culturelle et intellectuelle dans le Québec des $\mathrm{XvIII}^{\mathrm{e}}$ et $\mathrm{xIX}^{\mathrm{e}}$ siècles : quelques pistes de recherche», Revue d'histoire de l'Amérique française, 54,2 (automne 2000) : 271.

10. Janet Ajzenstat, "Durham and Robinson : Political Faction and Moderation», Journal of Canadian Studies/Revue d'études canadiennes, 25,1 (printemps 1990) : 24-38; Rod Preece, «The Myth of Red Tory», Canadian Journal of Political and Social Theory, 1 (1977) : 3-23 ; "The AngloSaxon Conservative Tradition", Canadian Journal of Political Science, 13,1 (mars 1980) : 3-20.

11. Georges Burdeau parle de la "conquête» et de «l'exploitation de la liberté», Le libéralisme (Paris, Seuil, 1979), 123, 131. Michel Branciard fait une distinction entre «libéralisme émancipateur» et «libéralisme politique post-révolutionnaire», Les libéralismes, d'hier à aujourd'hui (Lyon, Chronique sociale, 1987), 10, 29. Nancy L. Rosenblum discute du «moral idealism» et du «sober political modus vivendi», "Introduction» dans Liberalism and Moral Life (Cambridge, Harvard University Press, 1989), 5-6. 
individualisme ne signifie pas égoïsme. Le libéralisme est une théorie de l'individualisme social qui nourrit une préoccupation sincère envers le bien public. Parallèlement, l'homme est un être libre. Néanmoins, liberté n'est pas licence. Elle est encadrée par des balises. Elle est d'abord limitée par la raison incarnée dans les lois, condition de la liberté : là «où il n’y a point de loi, il n'y a point non plus de liberté ${ }^{12} »$. Le libéralisme repose donc sur une liberté individuelle responsable et tributaire de la loi ${ }^{13}$. Enfin, l'individu étant libre, il peut travailler à améliorer sa vie. La propriété apparaît alors comme un droit inviolable. L'inégalité de fait inhérente au libéralisme repose sur ce principe du libre destin : elle est le fruit de la liberté et du travail.

Par ailleurs, il «n’y a pas entre l’intervention de l'État et le libéralisme cette antinomie fondamentale que l'on a coutume d'admettre ${ }^{14} »$. Les libéraux savent que l'État constitue la condition de la liberté puisqu'il peut seul assurer sa mise en œuvre. Le libéralisme ne se dresse donc pas contre l'État mais contre son autoritarisme. Afin d'assurer la liberté et la sécurité des citoyens, les tenants du libéralisme ont développé certains mécanismes. Ils défendent l'importance de la constitution, véritable fondement de l'État. Ce système reconnaît le droit à l’opposition. Néanmoins, «l’État libéral ne connaît d'autre opposition que celle qu'il nomme "constitutionnelle" c'està-dire celle qui, s'exerçant dans le cadre des institutions, ne met pas en cause l'ordre constitutionnel établi ${ }^{15}$ ». Dans ce système, la souveraineté, source légitime du pouvoir suprême, doit commander respect et obéissance. Dans le contexte du libéralisme parlementaire britannique, elle appartient au Parlement et non au peuple.

Enfin, les libéraux proposent un partage des pouvoirs visant à empêcher l'État protecteur de se transformer en État oppresseur. Si Locke a discuté de l'existence de trois pouvoirs distincts, c'est Montesquieu qui a clarifié la théorie de la division des pouvoirs. Partant du principe que «pour qu'on ne puisse abuser du pouvoir, il faut que, par la disposition des choses, le pouvoir arrête

12. John Locke, Second traité du gouvernement civil (1690), VI, 57 (Paris, Garnier-Flammarion, 1992), 185 ; phrase citée par William Blackstone, The Commentaries on the Laws of England, I, 1 (New York, Garland Publishing, 1978) : 126. Pour Montesquieu, «la liberté est le droit de faire tout ce que les lois permettent», De l'esprit des lois, XI, iii (Paris, Garnier-Flammarion, 1979), 1 : 292.

13. Sur les limites du libéralisme, voir Uday S. Metha, "Liberal Strategies of Exclusion», dans Frederick Cooper et Ann Laura Stoler, dir., Tensions of Empire : Colonial Cultures in Bourgeois World (Berkeley, University of California Press, 1997), 59-86.

14. G. Burdeau, op. cit. 1979, 51.

15. Ibid., 221. 
le pouvoir ${ }^{16}$ ", il a défini trois pouvoirs distincts : le législatif, l'exécutif et le judiciaire. Si le pouvoir judiciaire est confié à des juges indépendants ${ }^{17}$, le pouvoir exécutif doit être l'apanage du monarque. Quant au pouvoir législatif, il appartient aux représentants de la "nation». Le principe représentatif sous-jacent est toutefois caractéristique de l'aversion que le libéralisme du $\mathrm{xVIII}^{\mathrm{e}}$ siècle $^{18}$ voue à la démocratie ${ }^{19}$ : seule une minorité privilégiée peut finalement voter. Cet élitisme vise à freiner les revendications populaires. De plus, ce libéralisme opte pour le bicaméralisme où une chambre héréditaire fait contre-poids à la chambre élue. De ce libéralisme se démarquera un second au $\mathrm{XIX}^{\mathrm{e}}$ siècle, plus soucieux de représentativité et pour lequel l'exécutif ne saurait être responsable que devant l'Assemblée des élus. Nous reviendrons sur cette distinction entre les deux libéralismes, car elle occupe une place centrale dans les discussions politiques au lendemain des rébellions.

\section{I.2 Nation et nationalisme}

Si le libéralisme est une idéologie complexe, le nationalisme l'est bien davantage. Les théories à son égard se sont multipliées ${ }^{20}$. Certains prétendent que la nation est le résultat de l'évolution du capitalisme, de l'industrialisation et du développement de l’imprimé. À partir de ce postulat, chacun y est allé de sa propre analyse. Benedict Anderson a conceptualisé la nation comme «[an] imagined political community». Ernest Gellner et Eric Hobsbawm ont mis l'accent sur la nécessité d'une unité politique et culturelle au sein du nationalisme. Quant à Rogers Brubaker, il a adopté une perspective semblable aux historiens réfléchissant au «State formation» canadien en traitant la nation non pas simplement comme la réification d'une idée, mais plutôt comme «[a] practical category, institutionalized form, and contigent event ${ }^{21} »$.

16. Montesquieu, De l'esprit des lois, XI, iv, $1: 293$.

17. Blackstone, Commentaries on the Laws of England, I, vii, $1: 259$; Montesquieu, De l'esprit des lois, XI, vi, 1 : 294; Smith, De la richesse des nations, IV, vii, section 3, $2: 224$; V, i, section 2, $2: 344-345$.

18. Si le terme «libéral» apparaît au XIX ${ }^{\mathrm{e}}$ siècle, les principes lui sont antérieurs. G. Burdeau, op. cit., (1979), 7.

19. Montesquieu, De l'esprit des lois, XI, vi, $1: 297$.

20. Pour une revue générale des théories, Gil Delannoi et Pierre André Taguieff, dir., Théories du nationalisme (Paris, Éditions Kimé, 1991), 324 p. Voir plus particulièrement l'article de Christophe Jaffrelot, «Les modèles explicatifs de l'origine des nations et du nationalisme. Revue critique ", 139-177.

21. Benedict Anderson, Imagined Communities. Reflections on the Origin and the Spread of Nationalism (Londres, Verso, 1991) (1983), 1; Ernest Gellner, Nations and Nationalism (Ithaca, Cornell University Press, 1983), 1; Eric Hobsbawm, Nations et nationalisme depuis 1780. Programme, mythe, réalité (Paris, Gallimard, 1992) (1990), 26; Rogers Brubaker, Nationalism Reframed. Nationhood and the National Question in the New Europe (Cambridge, Cambridge University Press, 1996), 7, 16, 21. 
Pour les trois derniers auteurs, le nationalisme a engendré les nations et non l'inverse, thèse qui va à l'encontre de la position défendue par Anthony $\mathrm{D}$. Smith et John A. Armstrong pour qui l'existence des nations a précédé le nationalisme ${ }^{22}$.

Théorie pour théorie, laquelle prime? Plutôt que de tenter de démontrer le bien-fondé de l'une sur l'autre, nous préférons adopter une approche plus empirique. Nous classifions les diverses formes de nationalisme selon la dichotomie désormais classique de civique versus ethnique. Cette opposition a été définie par plusieurs penseurs comme Hans Kohn, John Plamenatz, Gellner, Brubaker, Guy Hermet et Alain Finkielkraut ${ }^{23}$. Certes, elle n'est pas unanimement acceptée. Dans les années 1960, Élie Kédourie considérait que le nationalisme ne pouvait être qu’ethnique. Plus récemment, Stéphane Paquin s'est opposé à «cette dichotomie, fausse et manichéenne" parce qu'elle "n'est aujourd'hui pas très opérationnelle [...]», les identités étant plus complexes et fragmentées. De son côté, Michel Seymour a tenté de la transcender en proposant sa définition sociopolitique de la nation. Néanmoins, sa définition ressemble moins à un dépassement de la dichotomie qu'à la recherche d'une voie mitoyenne entre les deux conceptions. À toutes fins utiles, sa définition ressemble à celle de Gérard Bouchard qui recherche un équilibre entre territoire et langue. En fait, c'est peut-être Anthony D. Smith qui a le mieux résumé la situation. Sans remettre en cause les deux pôles de l'opposition, il a précisé que «every nationalism contains civic and ethnic elements in varying degrees and different forms ${ }^{24}$ ». Bref, pour bien comprendre une forme particulière de nationalisme, il faut déterminer les influences «civiques » et les influences «ethniques».

22. Anthony D. Smith, National Identity (Londres, Penguin Books, 1991), 227 p.; John Armstrong, Nations Before Nationalism (Chapel Hill, University of North Carolina Press, 1982), 411 p.

23. Hans Kohn, The Idea of Nationalism. A Study in its Origins and Background (New York, MacMillan, 1956), 4, 329 ss; Gellner, op. cit., 97-103 ; John Plamenatz, "Two Types of Nationalism», dans Eugene Kamenka, dir., Nationalism. The Nature and Evolution of an Idea (Londres, Edward Arnold, 1976), 23-36; Rogers Brubaker, Citizenship and Nationhood in France and Germany (Cambridge, Harvard University Press, 1992), 270 p.; Guy Hermet, Histoire des nations et du nationalisme en Europe (Paris, Seuil, 1996), 115-133; Alain Finkielkraut, La défaite de la pensée (Paris, Gallimard, 1987), 15-69.

24. Elie Kédourie, Nationalism (Londres Hutchinson University Library, 1966) (1960), 151 p. ; Stéphane Paquin, "Les théories explicatives de l'apparition des nations et du nationalisme», Bulletin d'histoire politique, 8,1 (1999) : 109; Michel Seymour, La nation en question (Montréal, L’Hexagone, 1999), 206 p. ; Gérard Bouchard, «La réécriture de l’histoire nationale du Québec. Quelle histoire? Quelle nation?», dans Robert Comeau et Bernard Dionne, dir., À propos de l'histoire nationale (Sillery, Septentrion, 1998), 134-139; Anthony Smith, op. cit., 1991, 13. 
Le nationalisme civique, ou patriotisme constitutionnel, est fondé sur l'attachement à un pays et à ses institutions. Dans ce cas, État et nation deviennent synonymes. Plus complexe est le nationalisme à caractère ethnique, parce que les notions d'État et de nation sont distinctes. Pour certains intellectuels, tout nationalisme demande une convergence entre l'État (unité politique) et la nation (unité culturelle). Cette position nous apparaît réductrice et plus étatiste que nationaliste dans la mesure où la souveraineté de l'État cesse d'être un moyen au service de la nation pour devenir une fin en soi. Théoriquement, les nationalistes veulent un État souverain parce que ce dernier est le moyen pouvant permettre la pleine réalisation de la nation. Mais que faire si l'État n'est pas le meilleur moyen pour assurer le développement de la nation? John Breuilly a alors précisé que si l'indépendance apparaît comme un élément important, il se peut qu'en certaines circonstances le nationalisme accepte un compromis ${ }^{25}$.

En fait, le nationalisme ethnique se divise en deux grandes tendances. Premièrement, il existe un nationalisme essentiellement culturel visant la préservation de la nation. Ce nationalisme implique l'aménagement d'un espace public réservé à la liberté, au développement et à l'expression de la culture nationale. Il s'avère plus ou moins politisé selon l'espace public demandé, l'attachement à un territoire ou l'autonomie réclamée. Ce nationalisme, ne revendiquant pas d'indépendance, exige minimalement la reconnaissance de quelques droits collectifs pouvant aller jusqu'à un gouvernement provincial fort $^{26}$. Les liens unissant le politique et le culturel étant distendus, ce nationalisme peut cohabiter à l'intérieur d'une fédération multinationale.

Le nationalisme politique, deuxième grande catégorie, connaît deux variantes importantes. La première considère qu'une culture doit s'incarner dans un État et incidemment vise à faire concorder frontières culturelles et étatiques ${ }^{27}$. Ce nationalisme ethnique politique peut être défensif ou agressif. Dans le premier cas, il recherche la survie de la nation, lutte contre la domination étrangère ou travaille à l'unité nationale. Dans l'autre

25. John Breuilly, Nationalism and the State (Manchester, Manchester University Press, 1982), 36.

26. Cette forme de nationalisme correspond au nationalisme culturel de A. D. Smith et à la version «ultra-faible» du nationalisme défini par Jean Leca. Anthony D. Smith, «Nationalism. A Trend Report and Bibliography», Current Sociology, 21,3 (1973) : 19; Jean Leca, "Nationalisme et universalisme», Pouvoirs, 57 (1991): 39.

27. Cette variante correspond à la version de Gellner, Hobsbawn, Kédourie ainsi qu’à la version «minimale» de Leca. E. Gellner, op. cit., 1 ; S. Hobsbawn, op. cit., 26; E. Kédourie, op. cit., 9 ; J. Leca, loc. cit., 39. 
cas, il tente de prendre de l'expansion aux dépens de ses voisins. Enfin, la variante «forte ${ }^{28}$ ", forme doctrinale du nationalisme, fait reposer la nation sur la nature. Cette vision, inspirée par Herder, place la nation au-dessus de tout. Par exemple, pour Georges Vacher de Lapouge : «l'individu est écrasé par sa race, et n'est rien. La race, la nation sont tout ${ }^{29}$.»

\section{I.3 De la juxtaposition des idéaux}

Certes, le libéralisme et le nationalisme se définissent idéologiquement comme antinomiques, le paradigme des droits individuels s'opposant à celui des droits collectifs. Ainsi, certains libéraux rejettent toutes considérations nationalistes parce que perçues comme ennemis de la liberté. Inversement, certains nationalistes rejettent les principes libéraux au nom de la nation ${ }^{30}$. La nation, et non plus la liberté, est vue comme naturelle. Cette forme de nationalisme soumet l'individu à une collectivité nationale considérée comme une réalité intrinsèque ne dépendant pas des individus.

Si le libéralisme et le nationalisme ne peuvent fusionner idéologiquement, les notions de liberté et de nation sont capables de cohabiter au sein d'un même discours. Si la primauté de l'individu constitue la base du libéralisme, cet individu est un être sociable. Un libéral ne peut donc nier l'importance de la collectivité ${ }^{31}$. Il peut même lui arriver d'octroyer des droits collectifs moyennant certaines conditions : «i) ces droits dérivent d'une manière ou d'une autre de droits individuels ou ii) ces droits ne s'opposent pas directement à un droit fondamental et sont au service du bien-être général des individus ${ }^{32}$ ». La collectivité peut donc s'avérer importante pour un

28. J. Leca, loc. cit., 39.

29. Georges Vacher de Lapouge dans L'Aryen. Son rôle social (1889-1890), cité par Pierre-André Taguieff, «Le nationalisme des nationalistes. Un problème pour l'histoire des idées politiques en France», dans Gil Delannoi et Pierre-André Taguieff, dir., Théorie du nationalisme (Paris, Kimé, 1991), 90.

30. Lord Acton apparaît comme l'exemple typique du libéral anti-nationaliste. Voir "Nationality" (1862) dans The History of Freedom and Other Essays (Londres, MacMillan, 1907), 207-300. De son côté, Johann Gottlieb Fichte, après 1806, représente ces nationalistes anti-libéraux, Discours à la Nation allemande (1807) (Paris, Alfred Costes, 1923), 245 p.

31. "The liberal view that only individuals count does not require liberals to be blind to the fact that group membership and shared cultural practices are important to individuals and play a significant role in helping many people to build valuable lives. » David Johnston, The Idea of a Liberal Theory. A Critique and Reconstruction (Princeton, Princeton University Press, 1994), 20.

32. François Blais, «Peut-on être libéral et nationaliste? Sur les conséquences normatives de l'individualisme moral dans le débat opposant libéralisme et nationalisme », dans François Blais, Guy Laforest et Diane Lamoureux, Libéralismes et nationalismes. Philosophie et politique (SainteFoy, Les Presses de l’Université Laval, 1995), 26. 
libéral si l'individu demeure au centre de la réflexion. Aussi longtemps que la collectivité sert les intérêts des individus, le libéralisme l'accepte.

Certains penseurs libéraux définissent la nation comme le cadre à l'intérieur duquel le libéralisme peut s'épanouir. John Stuart Mill, dans De la liberté, défend la primauté des droits individuels sur les droits collectifs ${ }^{33}$. Par la suite, il intègre certaines valeurs nationales à son discours. Ce libéralisme national est exprimé dans Considerations on Representative Government. Pour Mill, liberté et nationalité s'avèrent intimement liées, la nation apparaissant comme le moyen de la liberté : «Free institutions are next to impossible in a country made up of different nationalities ${ }^{34}$.» Il n'y a pas de déterminisme inaltérable chez Mill, seulement des considérations pratiques. L'existence de plusieurs langues au sein d'une même nation empêche la communication entre les divers groupes linguistiques et les met en présence de sources d'information différentes, ce qui nuit à la discussion et à la formation d'une opinion publique.

Si certains libéraux acceptent la nation sans l'ériger en fin, certains nationalistes empruntent des notions au libéralisme tout en faisant de la nation le cœur de leur préoccupation. Le degré de «libéralisme» peut varier d’un discours à l'autre. Néanmoins, ils se rejoignent tous dans l'idée que les principes empruntés au libéralisme sont au service de la nation. Le nationalisme d'Ernest Renan, développé dans Qu'est-ce qu'une nation? $(1882)^{35}$, se rattache à la tradition du nationalisme libéral français parce que la nation est vue comme le résultat de la volonté humaine et non comme celui d'un déterminisme quelconque. Pour Renan, «une nation est une âme, un principe spirituel. Deux choses, qui à vrai dire n'en font qu'une, constituent cette âme, ce principe spirituel. L'une est dans le passé, l'autre dans le présent. L'une est la possession en commun d'un riche legs de souvenirs; l'autre est le consentement actuel, le désir de vivre ensemble ${ }^{36}$.» Parce que la nation se base sur la volonté, son nationalisme est libéral.

En définitive, le libéralisme peut intégrer des valeurs nationales dans son discours. Inversement, le nationalisme ne rejette pas nécessairement les principes libéraux, bien que ces derniers soient soumis à l'intérêt national. Ce qui distingue les libéraux des nationalistes, c’est la hiérarchisation

33. John Stuart Mill, De la liberté (1859) (Paris, Gallimard, 1990), 61, 74.

34. John Stuart Mill, Considerations on Representative Government (1861) (Londres, J. M. Dent \& Sons, 1957), 361.

35. Ernest Renan, "Qu'est-ce qu'une nation?» (1882), dans Qu'est-ce qu'une nation? Et autres essais politiques (Paris, Presses Pocket, 1992), 36-56.

36. Ibid., 54. 
des principes. Pour le libéral, la nation est au service de l'individu. Pour le nationaliste, l'individu est au service de la nation. Chez un libéral, aucun déterminisme inaltérable n'est toléré. Chez un nationaliste, il peut y avoir certains déterminismes. Toutefois, plus le nationalisme a intégré de notions libérales, plus les déterminismes sont évanescents.

\section{2 - IDÉES ET IDÉOLOGIES CONCERNANT LES CANADAS}

De 1838 à 1840, dans les deux Canadas et en Grande-Bretagne, plusieurs idéologies coexistent. Pour certains, la défense de l'Empire britannique est centrale; pensons à lord Durham, lord Russell, le Conseil législatif du HautCanada et la Montreal Gazette. La Chambre d'assemblée du Haut-Canada articule un discours centré sur la communauté coloniale ${ }^{37}$. L'identité de cette dernière est ethnique parce que basée d'abord sur la langue anglaise. Elle est cependant libérale, car elle conçoit l'assimilation linguistique comme un outil d'intégration. Son sentiment d'appartenance est culturel dans la mesure où il ne revendique pas un pays en soi, mais le rattachement à l'Empire. Les Canadiens français aussi organisent leur argumentaire autour de la collectivité, mais nationale cette fois. Le nationalisme qui est articulé par Étienne Parent est culturel, en ce qu'il ne demande pas l'indépendance. En fait, dès 1831, le journaliste de Québec défend un programme précis qui vise à «maintenir tout ce qui parmi nous constitue notre existence comme peuple, et comme moyen d'obtenir cette fin de maintenir tous les droits civils et politiques qui sont l'apanage d'un pays anglais ${ }^{38}$ ». Parent vise la sauvegarde de la nationalité canadienne par l'utilisation du libéralisme politique britannique et du cadre impérial anglais. Il conserve cette position en $1838-1840^{39}$. Certes, l'historiographie s'est attardée à démontrer les variations dans son discours et ses changements de positions. Tel fut le cas parce que tous les auteurs nous ont offert une lecture linéaire des éditoriaux, c'est-à-dire une présentation des textes sans leur mise en rapport avec le contexte de leur rédaction ${ }^{40}$. Enfin, certains tra-

37. Il ne saurait être question d'une communauté nationale puisque la Chambre ne postule pas l'existence d'une nation haut-canadienne ni même canadienne.

38. Le Canadien, le 7 mai 1831.

39. Pour une explication plus détaillée, voir Michel Ducharme, "Quand la plume voile plus qu'elle ne dévoile. Le discours d'Étienne Parent (1838-1840)", dans Bernard Andrès et MarcAndré Bernier, dir., Portrait des arts, des lettres et de l'éloquence au Québec (1760-1840) (Québec/Paris, Les Presses de l’Université Laval/L'Harmattan, 2002), 385-397.

40. Marcel-Pierre Hamel, «Introduction» dans Marcel-Pierre Hamel, Le rapport Durham ([s.1.], Éditions du Québec, 1948), 51; Louis Nourry, La pensée politique d'Étienne Parent, 1831-1842, thèse de doctorat (histoire), Université de Montréal, 1971, 375-518; Denis Monière, Le développement 
vaillent à assurer une plus grande liberté et une plus grande autonomie aux coloniaux, pensons à Baldwin.

Malgré ces divergences, les discours ne sont pas aussi antagonistes que nous pourrions le croire. Notre propos vise à mettre en lumière les ressemblances entre tous ces discours. Il ne fait aucun doute que certains consensus se dessinent entre 1838-1840, laissant entrevoir la formation de l’État canadien.

\section{I Deux colonies, une métropole, un Empire}

L'idée de la séparation de la colonie d'avec l'Empire ou de l'annexion aux États-Unis continue à avoir quelques adeptes entre 1838 et 1840, à preuve la déclaration d'indépendance de Robert Nelson en février 1838 et la seconde rébellion du Bas-Canada en novembre. Plus tard, il y aura la crise annexionniste de 1849, les aspirations des Rouges du Canada-Est et de certains «Clear Grits » du Canada-Ouest ou même celle de Goldwin Smith à la fin du siècle. Toutefois, les rébellions de 1837 marquent la fin de la menace la plus sérieuse à l'intégrité de l'Empire, la seule tentative de renverser par la force l'ordre établi. Entre 1838-1840, hormis les rebelles, dont l'action s'avère finalement inefficace, personne ne conteste plus ouvertement l'appartenance des colonies à l'Empire. Le Canada en fera donc partie. L'acceptation du cadre impérial est renforcée par la conviction répandue parmi les coloniaux et même les métropolitains que les colonies doivent jouir d'une plus grande autonomie dans le cadre impérial. À Londres, lord Russell est clair : «the Governor must only oppose the wishes of the Assembly where the honour of the Crown, or the interest of the empire are deeply concerned ${ }^{41} \ldots$ ». De

des idéologies au Québec, des origines à nos jours (Montréal, Québec/Amérique, 1977), 155 ; Gérard Bergeron, Lire Étienne Parent. Notre premier intellectuel (Sainte-Foy, Presses de l'Université du Québec, 1994), 112 ; Janet Ajzenstat, The Political Thought of Lord Durham (Montréal, McGillQueen's University Press, 1988), 18; Stéphane Kelly, La petite loterie. Comment la Couronne a obtenu la collaboration des Canadiens français après 1837 (Montréal, Boréal, 1997), 183 ; «Introduction", dans Étienne Parent, Discours. Édition critique par Claude Couture et Yvan Lamonde (Montréal, Presses de l'Université de Montréal, 2000), 40-41. Seuls Philippe Reid et Jacques Monet semblent avoir évité le piège de la lecture linéaire des éditoriaux du Canadien. Le premier y parvient en ne traitant pas vraiment la période 1838-1840, le second, en remettant en cause la signification de ces éditoriaux. Voir Philippe Reid, «L'émergence du nationalisme canadien-français : l'idéologie du Canadien (1806-1842)», Recherches sociographiques, 21,1-2 (1980) : 20-53; Monet, op. cit., 27.

41. Lord John Russell to Poulett Thomson, le 14 octobre 1839 cité dans William Paul McClure Kennedy, dir., Documents of the Canadian Constitution 1759-1915 (Toronto, Oxford University Press, 1918), 524. À l'avenir, toutes les dépêches de lord Russell émanant de ce recueil, seul le numéro de la page d'où sont tirés les renvois sera indiqué. Voir aussi Lord John Russell on Canadian Affairs, 3 juin $1839,480$. 
son côté, lord Durham propose un partage des pouvoirs entre le gouvernement impérial et les administrations coloniales. Dans cette proposition, le gouvernement londonien conserve son pouvoir dans quatre domaines : la constitution, les affaires étrangères, le commerce impérial et international ainsi que la gestion et la distribution des terres publiques ${ }^{42}$. Aussi circonscrite qu'elle soit pour les administrations coloniales ${ }^{43}$, cette répartition des pouvoirs n'en est pas moins réelle.

Dans les colonies, Parent accepte le cadre impérial à la condition que l'Empire laisse à ces dernières une plus grande liberté. Selon lui, «il ne suffit plus pour l'Angleterre d'allonger la lisière, il faut qu'elle la coupe et nous laisse maître de nos mouvements ${ }^{44}$ ». De son côté, son collègue réformiste haut-canadien Baldwin articule une théorie originale ${ }^{45}$. Dans sa proposition de responsabilité ministérielle coloniale, Baldwin avance la scission de la souveraineté entre le Parlement impérial et le Parlement local. Le premier conserve la souveraineté en matière de politique extérieure, alors que le second agit souverainement en politique intérieure. Cette théorie est novatrice par rapport aux pratiques constitutionnelles impériales, pratiques ayant engendré la Révolution américaine ${ }^{46}$. Par sa proposition, Baldwin suggère une réforme fondamentale de l'Empire sans remettre son existence en cause.

Les tories coloniaux également acceptent l'idée d'une plus grande autonomie coloniale. Pour le Conseil législatif du Haut-Canada, une division stricte des pouvoirs est impensable, mais le gouvernement métropolitain doit se tenir à l'écart des questions internes autant que faire se peut ${ }^{47}$. Parallèlement, l'Assemblée législative veut que le gouverneur soit considéré comme un vice-roi. Se plaignant des changements fréquents à la tête

42. Sir Charles Lucas, dir., Lord Durham's Report on the Affairs of British North America, 2 : The Report (Oxford, Clarendon Press, 1912), 282. À l'avenir cite sous le nom de The Durham Report.

43. Voir Sir Charles Lucas, dir., Lord Durham's Report on the Affairs of British North America, 1 : Introduction (Oxford, Clarendon Press, 1912), 282 ; Gerald Craig, dir., Lord Durham's Report (Toronto, McClelland \& Stewart, 1963), vii; Ged Martin, The Durham Report and British Policy (Cambridge, Cambridge University Press, 1972), 59-60.

44. Le Canadien, le 14 février 1838; voir aussi Le Canadien, le 20 juin 1838.

45. Notre interprétation s'approche de celle de Paul Romney développée dans «From the Rule of Law to Responsible Government : Ontario Political Culture and the Origins of Canadian Statism ", Historical Papers, Canadian Historical Association (1988) : 86-119.

46. Ian R. Christie, Wars and Revolutions. Britain 1760-1815 (Londres, Edward Arnold, 1982), 107. Voir aussi Bernard Bailyn, The Ideological Origins of the American Revolution (Cambridge, The Belknap Press, 1967), 198-229.

47. Report of the Committee of the Upper Canada Legislative Council on Durham's Report (Toronto, R. Stanton, 1839), 13. 
du Colonial Office et du manque de fermeté des politiques londoniennes, l'Assemblée propose que le Colonial Office devienne un «conseil» (Board) dirigé par un membre du Cabinet et contenant des représentants des colonies. Plus encore, elle suggère que la colonie envoie des représentants à la Chambre des communes ${ }^{48}$. Bref, l'Assemblée législative désire pouvoir participer à la gestion de l'Empire. Seule la Montreal Gazette demande davantage d'interventions de la part de Londres. Paradoxalement, les tories bas-canadiens sont très sévères face aux décisions métropolitaines antérieures. Selon eux, la politique de conciliation adoptée par le gouvernement de Londres est directement responsable des malheurs de la colonie. Dans cette perspective, lord Glenelg et lord Gosford sont décrits comme des «impotent and incompetent rulers». Lord Gosford est également vu comme «the unparalleled of all imbecile Governors». Quant à la politique métropolitaine, elle provoque l'ire de l'organe tory. Selon lui, elle est caractérisée par «a spirit of unexampled weakness and folly», elle est qualifiée "d'imbécile» et d' "incohérente ${ }^{49}$ ». En fait, c'est le statut minoritaire des tories bas-canadiens qui explique qu'entre la domination canadienne-française et la domination impériale la seconde est perçue comme un moindre mal.

En somme, il devient évident dès 1838-1839 que l'appartenance à l'Empire est assurée. Toutefois, les liens entre la métropole et les colonies canadiennes, pour aussi solides et permanents qu'ils doivent être, sont tôt ou tard appelés à se distendre.

\subsection{L'organisation du pouvoir : un héritage libéral}

Le second consensus se situe au niveau politique. Traditionnellement, le Canada a été interprété par les historiens comme un État conservateur. Depuis quelques années, les historiens ont plutôt mis en lumière son aspect libéral. Pour y arriver, ils ont emprunté diverses approches telles que l'histoire institutionnelle, avec les adeptes du «State formation», l'histoire intellectuelle, avec Fernande Roy et Janet Ajzenstat, ou l'histoire politique, avec Gordon Stewart ${ }^{50}$. Grâce à ces travaux, l'apport du libéralisme dans

48. Report of a Select Committee of the House of Assembly on the State of the Provinces of Upper Canada and Lower Canada (Toronto, R. Stanton, 1838), 52.

49. Montreal Gazette, le 13 mars 1838; le 4 octobre 1838; le 24 avril 1838; le 25 septembre 1838 .

50. Fernande Roy, Progrès, Harmonie, Liberté. Le libéralisme des milieux d'affaires francophones à Montréal au tournant du siècle (Montréal, Boréal, 1988), 301 p. ; Janet Ajzenstat, op. cit., 18; loc. cit., 24-38; "The Constitutionalism of Étienne Parent and Joseph Howe», dans Janet Ajzenstat et Peter J. Smith, Canada's Origin. Liberal, Tory or Republican? (Ottawa, Carleton University Press, 1995), 209-232; Gordon T. Stewart, The Origins of Canadian Politics : A Comparative Approach (Vancouver, University of British Colombia, 1986), 129 p. 
la formation du Canada est reconnu. En fait, le libéralisme s'impose de manière définitive lors de la période 1838-1840. Par leur agitation inconstitutionnelle et illégale de 1837-1838 (le recours aux armes pour renverser un gouvernement n'était permis par aucune loi), les radicaux forcent la redéfinition de l'Amérique du Nord britannique. S'étant disqualifiés politiquement, ils ne peuvent participer à cet effort de réflexion. Certes, la seconde rébellion au Bas-Canada en 1838 ainsi que les raids des rebelles dans la province supérieure montrent que les radicaux n'entendent pas se faire oublier si facilement. Toutefois, leurs moyens d'action se situent en marge de la légalité et entraînent plus d'une trentaine de pendaisons dans les deux colonies. À la suite des rébellions, l'initiative politique retourne donc aux réformistes modérés et aux tories. L'échec des insurgés consacre le triomphe de la conception libérale de la politique partagée par les réformistes et les tories sur la conception républicaine des radicaux ${ }^{51}$.

Nous ne prétendons pas qu'il y ait identité idéologique entre modérés et tories ni même unanimité en leur sein. Néanmoins, tous acceptent que la société coloniale soit gérée suivant les principes libéraux. La différence réside dans leur compréhension de ces principes. Elle relève de la dichotomie recherche/conservation de la liberté. Pour les réformistes, des réformes s'avèrent essentielles pour permettre à la société de vivre dans un cadre libéral. Pour les tories, la liberté n'est pas vue comme l'objet d'une quête, mais comme un héritage à préserver.

Une variante importante du libéralisme pose ses assises sur la théorie des droits naturels. Selon cette théorie, les êtres humains possèdent des droits individuels inviolables. Chez Locke, ces droits naturels se résument en l'égalité, la liberté, la propriété, la sécurité et la vie. Au xviII siècle, Blackstone réduit ces droits au nombre de trois : «the right of personal security, the right of personal liberty, and the right of private property ${ }^{52}$ ». Dans la pratique coloniale, la théorie du droit naturel sert aussi bien les réformistes que les tories. Parent utilise la rhétorique des «droits naturels sacrés ${ }^{53}$ »,

51. Sur le républicanisme des radicaux, voir Louis-Georges Harvey, Importing the Revolution: The Image of America in French-Canadian Political Discourse, 1805-1837, thèse de doctorat (histoire), Université d'Ottawa, 1990, 508 p.; «Le mouvement patriote comme projet de rupture (18051837)", dans Gérard Bouchard et Yvan Lamonde, dir., Québécois et Américains. La culture québécoise aux XIX ${ }^{e}$ et XX $x^{e}$ siècles (Montréal, Fides, 1995), 89-112; "The First Distinct Society. French Canada, America and the Constitution of 1791 ", dans Janet Ajzenstat et Peter J. Smith, Canada's Origin. Liberal, Tory or Republican? (Ottawa, Carleton University Press, 1995), 79-108; J. E. Rea, «William Lyon Mackenzie Jacksonian?», Mid-America : An History Quarterly, L,3 (1968) : 223-235.

52. Blackstone, Commentaries on the Laws of England, I,1, $1: 129$.

53. Le Canadien, le 16 mars 1838. 
réclamant leur respect par Londres. La nation étant définie comme une propriété ${ }^{4}$, elle apparaît comme un droit naturel, ce qui dénote une déviation nationaliste de la théorie libérale. Les tories récupèrent aussi cette théorie. La Montreal Gazette parle de droits et de libertés sacrés définis comme "[the] rational liberty, with security of person and property ${ }^{55}$ ». Elle utilise la terminologie de Blackstone. C'est d'ailleurs au nom de ces droits inviolables des sujets britanniques qu'elle s'oppose aux prétentions démocratiques de la majorité : «We adhere to the principles of the Constitution, which prescribes justice to all, - which denies to a majority the right of tyrannizing over a minority — which secures to British subjects $[\ldots]$ the free and undoubted exercise of all their rights and privileges ${ }^{56} \ldots$ »

Dans la théorie libérale, les sujets sont légitimés de se révolter lorsque le pouvoir brime leurs droits ${ }^{57}$. Charles Dewey Day, juge-avocat du tribunal chargé de juger les patriotes, reconnaît ce droit dans une lettre publiée dans la Montreal Gazette ${ }^{58}$. Néanmoins, il nie que la situation au Bas-Canada ait permis une telle rébellion. L’Assemblée législative de la province supérieure adopte la même position ${ }^{59}$. De son côté, Parent intègre cette question dans le cadre d'une nation contractuelle. Citant Blackstone, il déclare que si une partie contractante ne respecte pas le contrat, l'autre partie se trouve déliée de ses obligations. Il définit incidemment la loyauté comme tributaire du respect des clauses du contrat ${ }^{60}$. Il reconnaît néanmoins que "de l'ordre actuel ne découlait pas ce degré d’oppression qui peut pousser un peuple au désespoir ${ }^{61} »$.

Lors d'une période troublée, si une conjuration menace la liberté, le pouvoir peut supprimer les droits et les libertés brièvement pour mieux les garantir de manière permanente. Pour Locke, l’exécutif peut «agir même quelquefois d'une manière contraire à des lois expresses de l'État,

54. Voir Le Canadien, le 16 mars 1838.

55. Montreal Gazette, le 20 février 1838. L’Assemblée législative de la colonie supérieure mentionne ces mêmes droits : "[...] the security of life, property, and that freedom which can only be called "glorious", when restrained by law ". Report of a Select Committee of the House of Assembly on the State of the Provinces of Upper Canada and Lower Canada (Toronto, R. Stanton, 1838), 41.

56. Montreal Gazette, le 8 février 1838.

57. Voir Locke, Second traité du gouvernement civil, XVIII, 207-210 ; XIX, 211-243, 295-327; William Blackstone, Commentaries on the Laws of England, IV,6, $4: 82$.

58. Montreal Gazette, le 17 février 1838.

59. Report of a Select Committee of the House of Assembly on the Political State of the Provinces of Upper Canada and Lower Canada (Toronto, R. Stanton, 1838), 6.

60. Le Canadien, le 10 janvier 1838.

61. Le Canadien, le 17 janvier 1838. 
si le bien public le requiert ${ }^{62} »$. Montesquieu mentionne aussi ce droit ${ }^{63}$. Enfin, Mill justifie l'autoritarisme du pouvoir dans ce cas : «I am far from condemning, in cases of extreme exigency, the assumption of absolute power in the form of a temporary dictatorship. [...] But its acceptance, even for a time strictly limited, can only be excused, if, like Solon and Pittacus, the dictator employs the whole power he assumes in removing the obstacles which debar the nation from enjoyment of freedom ${ }^{64}$.» En filigrane de ce commentaire théorique semble se dresser les leçons tirées de l'expérience canadienne. Mill connaît bien l'histoire des rébellions canadiennes pour les avoir commentées dans le London \& Westminster Review en 1838. Bien qu'il ait été initialement favorable à la cause des rebelles et se soit opposé à la suspension de la constitution, il défend la politique de lord Durham dès août 1838 pour en devenir un grand partisan à la fin de l'année ${ }^{65}$. Cette admiration perdure jusque dans son autobiographie ${ }^{66}$. Ainsi, en 1861, ce grand libéral ne décrie pas la suspension de la constitution et l'envoi d'un «dictateur» dans les colonies, mais légitime a posteriori cette politique puisqu'elle a permis la régénération de la politique impériale et l'introduction du principe du «internal self-government» dans les colonies.

À Londres, le gouvernement se sert de cette conception, permettant la suspension temporaire des libertés pour en assurer la pérennité, pour justifier sa politique. Lord Russell explique que «much as the suspension of constitutional government in Lower Canada is to be regretted, it will not be without a very considerable compensation, if, during the interval, arrangements should be maturely and wisely made for securing to the people at large the benefit of those social institutions from which, in former times, the thoughts of the local legislature were diverted, by the controversies

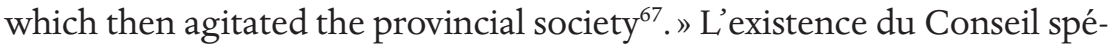

62. Locke, Second traité du gouvernement civil, XIV,164, 266.

63. Montesquieu, De l'esprit des lois, XI, vi, $1: 296$.

64. Mill, op. cit. (1861 b), 207.

65. John Stuart Mill, «Radical Party and Canada : Lord Durham and the Canadians», London \& Westminster Review (janvier 1838); «Lord Durham and His Assailants», London \& Westminster Review (août 1838); «Lord Durham’s Return", London and Westminster Review (décembre 1838). Ces articles sont reproduits dans Collected Works ofJohn Stuart Mill, VI; John M. Robson, dir., Essays on England, Ireland, and the Empire (Toronto/Londres, University of Toronto Press/Routledge \& Kegan Paul, 1982), 405-435, 437-443, 445-464.

66. John Stuart Mill, Autobiography (Londres, Longmans, Green, Reader, and Dyer, 1882), 216-217.

67. Lord John Russell to the Right Hon. C. Poulett Thomson, 7 septembre 1839, 519. 
cial ne trahit donc pas les fondements du libéralisme. Temporaire, il permet au gouvernement de continuer à gérer légalement une province dont la Chambre d'assemblée a menacé la constitution. En outre, l'administration du Conseil représente un apport essentiel à la mise en place des fondements libéraux de l'État à venir en minant définitivement les assises de la société pré-industrielle ${ }^{68}$. Lord Durham croit aussi la suspension de la constitution à la fois regrettable et nécessaire ${ }^{69}$. La Montreal Gazette considère que l'état d'anarchie dans lequel la province est plongée exige une solution énergique. Les responsables du journal défendent la mise en place d'un pouvoir autoritaire : "we have already expressed our opinion with respect to the necessity of the Autocracy under which we at present live in this Province, considering the anarchy and confusion in which we have been involved by the treasonable projects of the House of Assembly, supported by an ignorant and prejudiced constituency [...]. We know, that anarchy is always the precursor of despotism ${ }^{70}$.» Parent, rassure en mars 1838 par la nomination d'un enquêteur très libéral en la personne de lord Durham, accepte également la forme de gouvernement provisoire attribuée au Bas-Canada :

Longtemps avant ce jour, nous avons prédit que les agitateurs conduisaient ce pays au despotisme, car le despotisme fut toujours la conséquence inévitable de l'anarchie et de la licence, mais nous n'avions pas prévu que ce despotisme se présenterait sous une forme aussi bénigne et avec des espérances aussi encourageantes [...]. Accepter de bon cœur, et dans les sentiments qu'il parait nous être offert, l'état des choses provisoire qu'on nous présente; c'est le meilleur moyen de le voir promptement céder la place au gouvernement libre qu'on nous promet ${ }^{71}$.

Pour les libéraux, le bicaméralisme est longtemps apparu comme la norme. Montesquieu en a appelé à l'existence d'une chambre pour les privilégiés destinée à protéger les intérêts de la minorité "privilégiée» contre la majorité ${ }^{72}$. Outre cette considération élitiste qui vaut pour le système politique britannique en général aux $\mathrm{XVIII}^{\mathrm{e}}$ et $\mathrm{XIX}^{\mathrm{e}}$ siècles, il faut remarquer que le Conseil législatif bas-canadien a été conçu dans un cadre particulier :

68. Brian Young, «Positive Law, Positive State : Class Realignment and the Transformation of Lower Canada, 1815-1866», dans Allan Greer et Ian Radforth dir., Colonial Leviathan. State Formation in Mid-Nineteenth-Century Canada (Toronto, Toronto University Press, 1992), 50.

69. The Durham Report, 260.

70. Montreal Gazette, le 31 mars 1838.

71. Le Canadien, le 21 mars 1838.

72. Montesquieu, De l'esprit des lois, XI, vi, 1 : 297. 
il vise essentiellement à protéger la minorité anglophone privilégiée du pouvoir populaire francophone ${ }^{73}$.

Le gouvernement métropolitain, par la quatrième résolution Russell de mars 1837, entend préserver l'existence du conseil et rester maître des nominations tout en essayant de répondre aux vœux des représentants. Dans une dépêche adressée à Gosford datée du 6 mars 1837, Glenelg avertissait d'ailleurs le gouverneur que "the Legislative Council would be expanded to include a sizable number of members "holding opinions, in general, with those of the majority, but not concurring in their extreme demands"74». Même si lord Durham critique la composition du conseil et encourage sa réforme, tout en laissant à une commission le soin de proposer les changements à apporter à cette institution, il se félicite de son existence au Bas-Canada puisqu'il a sauvegardé les intérêts britanniques tout au long du conflit avec l'Assemblée législative ${ }^{75}$.

Chez les coloniaux, personne ne demande l'abolition du Conseil. Même Parent, qui aurait aimé le voir électif, reste coi sur cette question entre le 23 mars 1838 et le 16 décembre 1839. Baldwin, considérant que «the institutions of every Colony ought as nearly as possible to correspond with those of the Mother Country ${ }^{76}$ ", rejette l'idée de l'abolition pure et simple de la chambre haute de la Législature coloniale. Selon lui, «a second chamber of some kind has [...] been deemed essential to good government ${ }^{77}$ ». Quant aux tories, leur admiration pour la constitution britannique en fait des défenseurs acharnés du bicaméralisme dans les Canadas.

L'exercice pourrait se poursuivre, mais la conclusion demeurerait la même. Les grands principes libéraux se retrouvent indistinctement dans les écrits tory et réformistes. La souveraineté parlementaire n’est pas remise en cause, les institutions républicaines ne trouvent pas de défenseurs, l'élection du Conseil exécutif colonial ne sourit à personne, l'importance de l'éducation pour former la raison et permettre la liberté est

73. Voir Helen Taft Manning, "The Colonial Policy of the Whig Ministers 1830-1837 (II)", Canadian Historical Review, 33,4 (décembre 1952) : 351.

74. Cité dans Phillip A. Buckner, The Transition to Responsible Government. British Policy in British North America, 1815-1850 (Westport, Greenwood Press, 1985), 222. Peter Burroughs la paraphrase également dans The Canadian Crisis and British Colonial Policy 1828-1841 (Londres, Edward Arnold, 1972), 87.

75. The Durham Report, 82-83.

76. Baldwin to Glenelg, le 13 juillet 1836 (cité dans William Paul McClure Kennedy, Statutes, Treaties and Documents of Canadian Constitution, 1713-1929 (Londres, Oxford University Press, 1930), 336.

77. Ibid., 337. 
reconnue... Il est intéressant de constater que l'acceptation d'une chambre haute non élue, même s'il s'agit d'un pis aller pour Parent, montre bien l'attachement des intervenants à un libéralisme qui n'est pas encore essentiellement ni nécessairement démocratique. En fait, ce libéralisme fait primer la liberté des Modernes (protection des droits) sur la liberté des Anciens (participation à la vie publique) ${ }^{78}$. Dans cette perspective, l'appartenance à l'Empire ne cause pas de problèmes puisque, théoriquement, le principal rôle de l'État libéral (impérial ou non) se résume à assurer aux sujets la jouissance de leurs droits naturels.

Néanmoins, si tous acceptent le libéralisme et prônent son application dans les colonies, personne ne comprend les institutions britanniques de la même manière. Les tories défendent l'Acte constitutionnel comme la perfection faite constitution ${ }^{79}$. Ce faisant, ils adoptent une position relevant du libéralisme du xviII ${ }^{\mathrm{e}}$ siècle. Ce libéralisme pose ses assises, en théorie si ce n'est en pratique, sur la séparation des pouvoirs législatif et exécutif. Le premier appartient au Parlement; le second, à la Couronne. Si le monarque participe aux deux pouvoirs, la Couronne conserve encore une certaine indépendance. La prérogative de l'exécutif appartient au souverain et le rôle des conseils demeure officiellement plus discret ${ }^{80}$. De fait, lorsque Bagehot aborde les institutions de ce siècle, il considère que si la politique de l'exécutif s'avère tributaire de la volonté du Parlement, le choix des ministres demeure la prérogative de la Couronne ${ }^{81}$. C'est exactement ce que les tories coloniaux réclament. Ils demandent que le pouvoir royal dans la colonie choisisse librement ses conseillers eu égard à la volonté des élus. En contrepartie, la politique gouvernementale et le comportement des conseillers exécutifs sont directement soumis au contrôle de l'Assemblée ${ }^{82}$.

78. Sur la distinction entre la liberté des Anciens et la liberté des Modernes, voir Benjamin Constant, De la liberté des anciens comparées à celle des modernes. Discours prononcé à l'Athénée royal de Paris en 1819, reproduit dans Écrits politiques (Paris, Gallimard, 1997), 589-619; Madame de Staël, Des circonstances actuelles qui peuvent terminer la révolution et des principes qui doivent fonder la république en France (1798), I,3, édition critique de Lucia Omacini (Genève, Droz, 1979), 109-112.

79. Montreal Gazette, le 19 juin 1838, le 11 août 1838.

80. Voir Blackstone, Commentaries on the Laws of England, I,v (sur les conseils du roi) et vii (sur la prérogative royale).

81. Walter Bagehot, The English Constitution (1867) (Londres, Oxford University Press, 1955), 10.

82. Montreal Gazette, le 14 août 1838, le 7 septembre 1839; Report of the Committee of the Upper Canada Legislative Council on Durham's Report (Toronto, R. Stanton, 1839), 8. Sur l'ouverture des tories, voir Carol Wilton-Siegel, "Administrative Reform : A Conservative Alternative to Responsible Government», Ontario History, 78,2 (juin 1986) : 105-124. 
Le libéralisme sous-jacent à la suggestion de la responsabilité ministérielle se démarque de celui ayant inspiré la constitution de 1791. Cette revendication, théorisée par Baldwin et soutenue par Parent, s'inscrit dans la lignée du libéralisme institutionnel du $\mathrm{XIX}^{\mathrm{e}}$ siècle. En fait, les institutions britanniques de la seconde moitié du $\mathrm{xIx}^{\mathrm{e}}$ siècle ne sont plus tout à fait celles du $\mathrm{XVIII}^{\mathrm{e}}$ siècle : le raffinement de la pratique a modifié la théorie. Dans ce système, le cabinet dirige effectivement le pays sous l'autorité nominale de la Couronne. Par son interprétation, Baldwin anticipe l'analyse de Bagehot qui sépare les institutions constitutionnelles britanniques entre celles possédant un pouvoir nominal et celles au pouvoir effectif. Pour le célèbre commentateur de la constitution anglaise du XIx ${ }^{\mathrm{e}}$ siècle, «no one can approach to an understanding of the English institutions, or of others which, being the growth of many centuries, exercise a wide sway over mixed populations, unless he divides them into two classes. In such constitutions there are two parts [...]: first, those which excite and preserve the reverence of the population - the dignified parts, if I may so call them; and next, the efficient parts - those by which it, in fact, works and rules ${ }^{83}$." Baldwin propose que le représentant de la Couronne conserve son pouvoir de nomination, mais perde sa liberté de choix et son pouvoir de direction. Il propose que le gouverneur règne sans gouverner. Selon sa demande, le pouvoir exécutif doit appartenir à un conseil exécutif responsable devant la Chambre d'Assemblée sous l'autorité de la Couronne. Bref, sa proposition suit les grandes lignes de la constitution britannique, les devançant peut-être ${ }^{84}$, sans jamais les trahir.

Entre ces extrêmes, les réformistes métropolitains tentent une transition tout en douceur. Entre lord Durham et lord Russell, les ressemblances s'avèrent plus fondamentales que les différences. La «responsabilité» que Durham propose dans la lettre, lord Russell entend l'appliquer dans l'esprit ${ }^{85}$ tout en niant la concéder pour des raisons pratiques ${ }^{86}$. Durham précède simplement Russell dans sa compréhension des liens entre Empire et liberté, d'où

83. Bagehot, op. cit., 3-4.

84. Buckner, op. cit., 3-20. Sur la question du gouvernement responsable colonial, voir aussi Ajzenstat, op. cit., 59-60, 61, 63.

85. "The importance of maintaining the utmost possible harmony between the policy of the legislature and of the government admits of no question, and it will of course be your anxious endeavour to call to your counsels and to employ in the public service those persons who, by their position and character, have obtained the general confidence and esteem of the inhabitants of the province. » Lord John Russell to the Right Hon. C. Poulett Thomson, le 7 septembre 1839, 518.

86. Lord John Russell to Poulett Thomson, le 14 octobre 1839, 522-523. 
les divergences pratiques entre les deux hommes. Dans cette vision métropolitaine, l'indépendance de la prérogative royale est protégée, véritable legs du XviII ${ }^{\mathrm{e}}$ siècle. Même Durham entend confier la direction des colonies à un gouverneur cumulant les rôles de représentant de la Couronne et de premier ministre ${ }^{87}$. Ce faisant, la prérogative demeure intacte puisque le gouverneur conserve son droit de choisir ses conseillers et de les diriger. Toutefois, le représentant de la Couronne doit procéder aux nominations en respectant les vœux de l'Assemblée. Bref, les positions de lord Russell et de lord Durham laissent entrevoir une vision transitoire et pragmatique entre le libéralisme du $\mathrm{xVIII}^{\mathrm{e}}$ siècle et celui du XIx ${ }^{\mathrm{e}}$ siècle.

\subsection{Une nation ou des nations?}

La question coloniale canadienne se complique lorsque vient le temps d'intégrer les valeurs nationales dans les discours. Néanmoins, certaines perspectives dominantes se dégagent quant à la question nationale. Pour les tories coloniaux, des facteurs culturels (langue, coutumes, manières anglaises) et politiques (loyauté à l'Empire, à la constitution et aux lois) participent à la définition de la nation ${ }^{88}$. Les Canadiens, ne parlant pas anglais, possédant des lois particulières, cultivant des traditions distinctes et s'étant montrés déloyaux envers la constitution, ne font pas partie de cette nation. La nation étant source d'égalité, les Canadiens français ne peuvent être considérés comme des égaux. Leur assimilation apparaît comme un préalable à la représentation. Cette assimilation leur procurera l'égalité ainsi que l'occasion de comprendre et d'apprécier la liberté. La perspective tory demeure tout de même libérale dans le sens où l'assimilation est perçue comme le moyen d'intégrer les Canadiens français dans la nation britannique d'Amérique du Nord ${ }^{89}$.

Sur la question nationale, lord Durham partage les vues des tories. Dans cette optique, Marcel-Pierre Hamel a raison de soutenir que «le politique reconnu comme le plus libéral de la Grande-Bretagne [Durham] se trouva être le plus tory à l'égard des Canadiens français ${ }^{90} »$. En fait, Durham aborde

87. The Durham Report, 155, 279-280, 327 ; Buckner, op. cit., 259.

88 Sur la définition tory de la nation, voir par exemple la Montreal Gazette, le 6 février 1838, le 17 février 1838 , le 16 juin 1838 .

89. Par exemple, les éditeurs de la Montreal Gazette ne contestent pas le fait que les Canadiens français aient le droit de jouir de tous les droits des sujets britanniques. Néanmoins, ils concluent que leur assimilation aux manières et à la langue anglaises doit précéder la jouissance de ces droits. Le contraire ne peut mener qu'à la destruction de la constitution. Montreal Gazette, le 24 février 1838 et le 16 novembre 1839.

90. Hamel, loc. cit., 40. 
la question nationale selon deux angles. Il perçoit d'abord la réalité à travers ses préjugés libéraux. Il décrit l'infériorité des Canadiens français au niveau économique par leur désintérêt face au commerce ${ }^{91}$. Or le commerce est garant de l'ordre et du bon gouvernement pour les pères du libéralisme ${ }^{92}$. N'étant pas intégrés au commerce et n'ayant pas adopté des politiques économiques libérales, les Canadiens français demeurent des «brigands» au sens où Montesquieu entend ce mot $^{93}$. D'ailleurs, Durham donne aux Canadiens les qualités des peuples barbares, c'est-à-dire non liés au commerce : «they are mild and kindly, frugal, industrious and honest, very sociable, cheerful and hospitable, and distinguished for a courtesy and real politeness, which prevades every class of society ${ }^{94}$.» Du côté politique et social, les Canadiens français se complaisent dans leurs vieilles institutions. $S$ 'opposent à eux les anglophones des deux Canadas, supérieurs parce que passés maîtres dans l'art de commercer et incidemment de vivre en liberté ${ }^{95}$.

Parallèlement, Durham voit un problème pratique à la cohabitation de deux cultures dans un même État. Les problèmes de communication entre les deux communautés rendent impossible leur collaboration : «The difference of language produces misconceptions yet more fatal even than those which it occasions with respect to opinions; it aggravates the national animosities, by representing all the events of the day in utterly different lights ${ }^{96}$.» La perspective de Durham n'est pas très différente de celle

91. The Durham Report, 30.

92. Pour Montesquieu, "c'est presque une règle générale que, partout où il y a des mœurs douces, il y a du commerce; et que, partout où il y a du commerce, il y a des mœurs douces [...] l'effet naturel du commerce est de porter à la paix». De l'esprit des lois, XX,i, 2 : 9-10. Smith n'en pense pas moins : «le commerce et les manufactures introduisent par degrés un gouvernement régulier et le bon ordre, et avec eux la liberté et la sûreté individuelle, parmi les habitants de la campagne qui avaient vécu jusqu'alors dans un état de guerre presque continuel avec leurs voisins, et dans une dépendances servile avec leurs supérieurs...» Adam Smith, De la richesse des nations, III,4, $1: 502$.

93. "La privation totale du commerce produit, au contraire, le brigandage [...]. L'esprit n'en est point opposé à de certaines vertus morales : par exemple, l'hospitalité, très rare dans les pays de commerce, se trouve admirablement parmi les peuples brigands." Montesquieu, De l'esprit des lois, XX,i, $2: 10$.

94. The Durham Report, 30. Il est intéressant de noter qu'Alexis de Tocqueville partage l'analyse libérale de Durham sur les qualités candiennes-françaises : «[...] leurs mœurs sont douces et leur caractère serviable. Le peuple est en général plus moral, plus hospitalier, plus religieux qu'en France.» Alexis de Tocqueville, "Cahier alphabétique A, 2 septembre 1831 », dans Oeuvres complètes. Édition définitive publiée sous la direction de J.-P. Mayeur, V : Voyage en Sicile et aux États-Unis (Paris, Gallimard, 1957), 218.

95. The Durham Report, 26, 35.

96. The Durham Report, 40-41. 
de Mill à cet égard : "Among a people without fellow-feeling, especially if they read and speak different languages, the united public opinion, necessary to the working of representative government, cannot exist ${ }^{97}$." Toutefois, il double ce problème pratique d'un élément plus fondamental. Selon lui, la division linguistique crée au sein de l'État une division insurmontable entre les deux groupes, étant donné que la langue reflète la mentalite : «those who have reflected on the powerful influence of language on thought, will perceive in how different a manner people who speak in different languages are apt to think ${ }^{98}$.» Deux langues ne peuvent conséquemment coexister dans un même État. Si Adam Smith s'est penché sur la question des langues, il n'a jamais tenu de pareils propos ${ }^{99}$. En fait, cette assertion se compare davantage aux arguments de Fichte pour qui «le langage a plus d'influence sur les hommes que les hommes n'en ont sur le langage $^{100}$ \%. Sa vision du problème bas-canadien est donc à la fois libérale et nationale. Pour arriver à l'uniformité culturelle, qui seule peut permettre la liberté, Durham adopte la même vision que les tories : l'assimilation des francophones. Il ne souhaite aucunement avilir ladite société, mais bien la coopter dans la race anglaise. Cette solution s'avère libérale parce qu'elle ne sous-tend aucun déterminisme inaltérable. Par l'assimilation, les Canadiens français vont se débarrasser de leur lourd héritage et sortir de leur infériorité. Cette analyse laisse présager celle de Mill pour qui les races inférieures doivent être assimilées aux races supérieures, cette assimilation se faisant à l'avantage des assimilés qui peuvent ainsi évoluer ${ }^{101}$.

À Londres, la perspective est moins tranchée. En 1837-1838, la politique métropolitaine ne semble pas avoir nourri des visées strictement nationales. Il suffit de suivre l'évolution de la composition du Conseil législatif du BasCanada pour s'en convaincre. En mars 1837, les Canadiens français comptent pour moins de $40 \%$ du conseil (13 conseillers sur 34). Grâce aux nominations du 22 août de la même année, cette proportion augmente à

97. Mill, op. cit. (1861 b), 361.

98. The Durham Report, 40.

99. Voir Adam Smith, "Considerations Concerning the First Formation of Languages, and the Different Genius of Original and Compounded Languages ", dans Adam Smith, The Theory of Moral Sentiments (Londres, Henry G. Bohn, 1853) (Reproduction de New York, Augustus M. Kelley, 1966), 505-538.

100. Fichte, op. cit. (1807), 54. Il poursuit en disant que «ce n'est donc pas eux [les hommes] qui forment la langue, c'est la langue qui les forme». Ibid., 60.

101. Mill mentionne toutefois que, dans le cas où l'assimilation s'avérerait impossible, la séparation des deux nations ou leur fédération constitue la seule solution. Sur cette question, voir Mill, op.cit. (1861 b), 359-366. 
$46 \%$ (soit 19 conseillers sur 41 ). Enfin, selon les indications fournies par lord Gosford, sur les 40 conseillers d'octobre 1837, seuls 31 sont actifs et participent aux délibérations. De ce nombre, 18 sont Canadiens français $(58 \%)^{102}$. Bref, à la suite des Résolutions Russell, la proportion de Canadiens français au conseil est significativement majorée. En fait, Londres fait des efforts pour nommer plus de conseillers francophones depuis 1828. Sur 33 nominations faites depuis cette date, 21 sont canadiennes-françaises, soit $64 \%$ des nominations. En août 1837, des 10 nouveaux conseillers, 7 sont Canadiens français, soit $70 \%$ des gens promus. Certes, ces nominations se font en fonction d'objectifs politiques. Toutefois, elles indiquent que Londres ne déconsidère pas systématiquement les francophones avant les rébellions. D’un autre côté, si seule la constitution bas-canadienne disparaît à la suite des troubles de 1837, la survie de la Législature haut-canadienne s'explique non par des considérations nationales mais plutôt par un contexte politique différent. L'Assemblée bas-canadienne, étant composée d'une majorité patriote, ne peut être convoquée. Rebelle elle a été, disgraciée elle ne peut qu'être. Dans la province anglophone, les deux chambres de la Législature sont contrôlées par des tories loyaux à l'Empire. La Législature haut-canadienne peut donc participer à la gestion de la crise.

La question nationale prend une nouvelle dimension à la suite des rébellions. L'union signifie la perte d'une enclave territoriale pour les Canadiens français, la marginalisation officielle de leur langue au sein des nouvelles institutions coloniales et la surreprésentation anglophone à l'Assemblée. Toutefois, aucune instruction envoyée aux gouverneurs Durham et Sydenham ne traite explicitement de la question nationale et aucune théorie basée sur des arguments nationaux n'est avancée. Peut-être les dirigeants de Londres pensent-ils qu'une population culturellement homogène leur faciliterait la tâche. Même en prenant cette hypothèse, force nous est de constater que les politiques suivies manquent de vigueur et qu'elles ne peuvent être efficaces étant donné le poids démographique des Canadiens français et leur concentration sur le territoire. Ainsi, dès 1842, lorsque les tories de Robert Peel seront au pouvoir, le ministre des colonies lord Stanley spécifiera au gouverneur Charles Bagot : "You cannot too early, and too distinctly give

102. Pour les données, voir Joseph Desjardins, Guide parlementaire historique de la province de Québec 1792-1903 (Québec, [s.é.], 1902), 395 p.; Earl of Gosford, Copy of a Despatch from the Earl of Gosford to lord Glenelg, Castle of St-Lewis, Quebec, 19 October 1837 reproduit dans British Parliamentary Papers - Colonies - Canada, 9 : Reports, Correspondence, Returns and Other Papers Relating to Canada and the Earl of Durham's Appointment there 1837-1838 (Shannon (Irlande), Irish University Press, 1969). 
it to be understood that you enter the Province with the determination to know no distinctions of National origin, or Religious Creed ${ }^{103}$.» Stanley clarifiera également sur quelle base le gouverneur doit traiter les coloniaux : "The only Passport to your favor will be, Loyalty to the Queen, attachment to British connexion, $\&$ an efficient and faithful discharge of Public Duty $^{104}$. " En fait, même si le gouvernement métropolitain adopte une vision nationale, il ne prend pas les moyens de transformer la réalité. Dans cette situation, les Canadiens français doivent prouver leur loyauté à l'Empire, moyen de retrouver la confiance de Londres.

Dans les colonies, les réformistes développent des visions plus ouvertes à une coopération multinationale. Baldwin ne définit jamais ses concitoyens en termes nationaux. L'attachement à son "pays" (country) rappelle celui nourri par Smith envers ses institutions politiques et ses concitoyens ${ }^{105}$. Malgré l'emploi du mot «race», Baldwin définit ses compatriotes en termes exclusivement patriotiques, ne faisant appel qu'à des considérations strictement politiques :

Your Lordship [lord Durham] must adapt the Government to the genius of the people [...]. It is the genius of the English race in both hemispheres to be concerned in the Government of themselves.- I would ask Your Lordship, would the people of England endure any system of Executive Government over which they had less influence than that which at present exists? Your Lordship knows they would not. Can you then expect the people of these colonies with their English feelings and English sympathies to be satisfied with less? ${ }^{106}$

Cette vision intègre tous les Haut-Canadiens au sein d'une même collectivité. La collaboration des coloniaux dans la construction de la colonie doit se fonder sur des considérations essentiellement politiques, laissant les facteurs culturels au second plan. Ainsi, la collaboration des réformistes des deux Canadas dans le cadre de l'union peut se produire à la condition que les Canadiens français acceptent le cadre impérial et les institutions libérales britanniques.

103. Stanley's Instructions to Bagot, 8 octobre 1841, tiré de William Paul McClure Kennedy, dir., Statues, Treaties and Documents of Canadian Constitution, 1713-1929 (Londres, Oxford University Press, 1930), 459.

104. Stanley's Instructions to Bagot, 8 octobre $1841,459$.

105. Smith, The Theory of Moral Sentiments (1759), IV,ii, 2 (New York, Augustus M. Kelley, coll. «Reprints of Economic Classics», 1966), 339.

106. Baldwin to Durham, le 23 août 1838, 368. L'expression «English feelings and English sympathies» ne peut faire référence à une sorte de nationalisme anglais dans le contexte réformiste canadien : Baldwin est d'origine irlandaise. Sur l'idée d' Englishness», voir Linda Colley, Britons. Forging the Nation 1707-1837 (New Haven, Yale University Press, 1992), 105-117. 
C’est Parent qui redéfinit le nationalisme canadien-français de manière à rendre possible la coopération avec les réformistes anglophones. Rejetant l'aspect politique du nationalisme patriote, l'indépendance du Bas-Canada n’apparaissant plus réalisable, Parent définit un nationalisme culturel acceptant le cadre impérial. Délaissant en outre les idées républicaines développées par les Patriotes, il réintègre le nationalisme canadien dans un cadre politique libéral. Le nouveau nationalisme canadien-français cherche à assurer la survie de la nation canadienne définie dans l'Acte de Québec ${ }^{107}$ par l'acquisition d'institutions libérales. La position de Parent est claire :

Que le peuple Canadien soit maintenu dans ses droits naturels, civils et politiques, qu'on le laisse se développer librement sur son sol, qu'aucune prétention à la domination oligarchique ne soit favorisée, et le drapeau Britannique flottera longtemps encore triomphant et vénéré au haut de nos citadelles : Nationalité Canadienne et Souveraineté Britannique, telle est notre devise politique; la justice, l'intérêt et l'honneur de la Grande-Bretagne exigent qu'elle n'en ait pas d'autre ${ }^{108}$.

Devenu culturel et libéral, le nationalisme canadien-français devient compatible avec les visées réformistes haut-canadiennes. D’ailleurs, Parent, dans l'expectative de l'union des Canadas, se réconforte à l'idée qu'elle permettra probablement le rassemblement des forces réformistes et ainsi favorisera l'avènement du gouvernement responsable ${ }^{109}$. Au Haut-Canada, Francis Hincks, organisateur réformiste et rédacteur du Examiner de Toronto, est le premier à entrevoir la possibilité d'une alliance avec les Canadiens de langue française. En 1839, il entre en contact avec Louis-H. La Fontaine ${ }^{110}$ pour tisser des liens entre les deux groupes réformistes. L'union des réformistes signifie le début de la coopération entre deux groupes nationaux distincts unis par des objectifs politiques communs.

\section{CONCLUSION}

Les rébellions rendant nécessaire la redéfinition politique de l'Amérique du Nord britannique, tous les politiciens et les intellectuels non discrédités par la violence insurrectionnelle et intéressés par l'avenir des Canadas proposent

107. «Si l'on veut avoir une idée encore plus précise de ce que les Canadiens appellent leur nationalité, on la trouvera dans l'Acte Impérial de la $14^{\mathrm{e}}$ Geo III ch. 83, qui rétablit les anciennes lois du pays, et notamment dans la section $4^{\mathrm{e}}$, la $5^{\mathrm{e}}$ et la $8^{\mathrm{e}}$ Section de cet Acte [...]». Le Canadien, le 23 février 1838.

108. Le Canadien, le 2 février 1838.

109. Voir Le Canadien, le 8 juin 1838, le 8 mai, 13 mai, 3 juillet, 17 juillet et 23 décembre 1839.

110. Monet, op. cit., 47-48. Voir aussi Mason Wade, Les Canadiens français de 1760 à nos jours, 1 : 1760-1914 (Ottawa, Cercle du Livre de France, 1963), 255-256. 
leur solution. Malgré la diversité idéologique, certains consensus se dégagent. Les Canadas continueront à faire partie d'un Empire britannique évoluant vers la décentralisation. Au point de vue politique, la question fondamentale n'est plus de savoir si les Canadas seront libéraux ou républicains, mais de quel type de libéralisme ils vont s'inspirer : celui du XviII siècle ou celui du $\mathrm{XIX}^{\mathrm{e}}$. Les Canadas se définissant comme des colonies, leur sort dépend d'abord de la volonté du gouvernement métropolitain. En fait, ce dernier tente une transition entre ces deux libéralismes dès la décennie 1830. Helen Taft Manning a même soutenu que, durant cette décennie, les ministres whigs orientent leur politique "as rapidly as they could in the direction of colonial self-government ${ }^{111}$ ». Ce libéralisme de transition apparaît aussi bien dans le rapport de lord Durham que dans les dépêches de lord Russell. Ainsi, le libéralisme du XVIII ${ }^{\mathrm{e}}$ siècle ne peut triompher, les métropolitains cherchant à s'en éloigner. Quant à la durée de la transition vers celui du XIX ${ }^{\mathrm{e}}$ siècle, elle est tributaire des questions nationale et impériale.

D’une part, les métropolitains doivent définir l'Empire de telle sorte que les principes de liberté et d'Empire ne soient plus antagonistes. Lord Durham propose de défendre l'Empire par la liberté. Il rejoint la vision des réformistes coloniaux sur ce point. Il se démarque de son ami ministre, lord Russell, qui ne sait comment unir les valeurs impériales et libérales dans un même discours et s'oppose aux tories qui défendent l'Empire parfois aux dépens de la liberté. Il rejoint toutefois les réformistes dans leur vision d'un Empire uni par la liberté. Cette vision unissant Empire et liberté va être reprise en Grande-Bretagne par les Peelites dans la décennie suivante ${ }^{112}$. D'autre part, la transition deviendra possible lorsque le gouvernement britannique aura la certitude que la survie des Canadiens français ne menace plus celle de l'Empire. Si l'analyse ethnique des tories et de Durham n'offre guère d'espoir aux Canadiens français, la vision de Londres s'avère moins menaçante. Plus encourageante encore, l'analyse purement politique des réformistes haut-canadiens permet d'entrevoir une collaboration politique là où la diversité culturelle ne pose aucun problème. En fait, les Canadiens français sont responsables de leur sort. En repositionnant leur nationalisme sur le plan culturel et en faisant leurs les revendications pour des institutions libérales, ils peuvent peu à peu regagner la confiance de Londres. Cette «confiance» sera rétablie durant la décennie 1840. À ce moment, la transition vers le libéralisme du $\mathrm{xIx}^{\mathrm{e}}$ siècle pourra être complétée.

111. Helen Taft Manning, "The Colonial Policy of the Whig Ministers 1830-1837 (I)», Canadian Historical Review, 33,3 (septembre 1952) : 203.

112. Susan H. Farnsworth, The Evolution of British Imperial Policy During the Mid-Nineteenth Century: A Study of the Peelite Contribution, 1846-1874 (New York, Garland Publishing, 1992), 438 p. 